Artigo / Article

\title{
O xigenação extracorpórea por membrana e alterações hematológicas em estudo experimental
}

Aírton C. Moscardini
Moacir F. Godoy
Domingos M. Braile $^{2}$
José M. P. de Godoy
Marcelo J. Soares
Antonio C. Brandi
Serginando L. Ramin
A oxigenação extracorpórea por membrana é uma técnica de suporte cardiopulmonar prolongado, com finalidade de auxiliar o pulmão e/ ou o coração, quando os mesmos entram em falência não responsiva aos tratamentos convencionais não invasivos. 0 objetivo do presente estudo foi avaliar as alterações hematimétricas durante 12 horas de ECMO em estudo animal.

Foram estudados 11 ovinos, da raça Santa Inês, sendo seis machos e cinco fêmeas, com peso entre 5,4 e $15 \mathrm{~kg}(12,2 \pm 3,1 \mathrm{~kg})$. Nos cinco primeiros animais, o prime do circuito extracorpóreo foi feito com solução de Ringer com lactato de sódio (Ringer-Lactato Glicolabor - Ribeirão Preto - SP) e solução de gelatina a 3,5\% (Hisocel - Campinas - SP), na proporção de $2: 1$, no total de $750 \mathrm{ml}$. No sexto animal foi diminuído o volume do prime para $450 \mathrm{ml}$, sendo $250 \mathrm{ml}$ de solução de Ringer com lactato de sódio e $200 \mathrm{ml}$ de solução de gelatina a 3,5\%. Nos cinco últimos experimentos (casos 7 a 11), empregou-se $250 \mathrm{ml}$ de sangue fresco total anticoagulado com citrato de sódio e $200 \mathrm{ml}$ de Ringer com lactato de sódio. Os exames realizados foram: hematócrito, hemoglobina, leucócitos, plaquetas, albumina e globulina. No estudo estatístico foram empregados os testes de Mann-Whitney e Análise da Variância e, no caso desteúltimo demonstrar variação significativa $(p<0,05)$, aplicou-se 0 teste de Tukey.

Foi detectada redução em todos elementos analisados e com diferença estatística entre o grupo 1 e 2 no hematócrito e leucócitos. A oferta excessiva de volume desencadeou alterações hematimétricas em estudo experimental utilizando a ECMO em carneiro.

Rev.bras. hematol.hemoter.,2002,24(2):97-104

Palavras-chave: Oxigenação extracorpórea, alterações hematológicas

\section{Introdução}

A oxigenação extracorpórea por membrana é efetiva para os casos de assistência circulatória numa emergência, e pode representar uma opção de salvamento da vida do paciente (1). É uma técnica de suporte cardiopulmonar prolongado, com finalidade de auxiliar o pulmão e/ou o coração, quando os mesmos entram em falência não responsiva aos tratamentos convencionais não invasivos (2). Esta técnica é conhecida também pela sigla ECMO (Extracorporeal Membrane Oxygenation).

Ela é uma opção de tratamento, porém, apresenta alguns riscos de complicações e dentre elas podemos citar as alterações hematológicas.

A hemólise é uma delas e permanece como um dos mais sérios problemas durante bypass cardiopulmonar, oxigenação extracorpórea por membrana e o suporte cardiopulmonar

1 - Depto Pediatria da Faculdade de Medicina de São José do Rio Preto (FAMERP)

2 - Depto de Cardiologia e Cirurgia Cardiovascular da Faculdade de Medicina de São José do Rio Preto (FAMERP)

Correspondência para: Aírton Camacho Moscardini

Av. Brigadeiro Faria Lima, 5416. São José do Rio Preto. SP. CEP 15090-000. 
percutâneo. Entretanto, as características destas hemólises não são bem definidas (3). Em um dos estudos foi demonstrado que o prolongamento da ECMO desencadeou a trombose no circuito seguida de hemólise (4).

Outra alteração detectada foi 0 desenvolvimento de resposta inflamatória (5). A ECMO induz a ativação leucocitária e liberação de citocina. Esta reação pode, como trauma adicional, deteriorar ainda mais a situação já grave do paciente (6). Foi observada a redução do número de leucócitos $(7,8)$, porém a capacidade fagocitária foi mantida (9).

Nestes anos algumas alterações técnicas foram incorporadas a ECMO com a finalidade de reduzir a prevalência de algumas das complicações. Uma delas foi a utilização de dupla via de drenagem da veia jugular interna para aumentar o retorno venoso e a ultrafiltração como recurso para redução da volemia $(10,11)$.

0 objetivo do presente estudo foi avaliar as alterações hematimétricas durante 12 horas de ECMO em estudo animal.

\section{$M$ aterial e métodos}

Foram estudados 11 ovinos, da raça Santa Inês, sendo seis machos e cinco fêmeas, com peso entre 5,4 e $15 \mathrm{~kg}(12,2 \pm 3,1 \mathrm{~kg})$. Como medicações pré-anestésica empregou-se cloridrato de cetamina (Ketalar - Parke Davis- Guarulhos -SP) e cloridrato de 2-(2,6-xilidino)-5,6-dihidro-4H-1,3-tiazina (Rompum - Bayer - Porto Alegre - RS), nas doses de $5 \mathrm{mg} / \mathrm{kg}$ e $4 \mathrm{mg} / \mathrm{kg}$, respectivamente, por via intramuscular.

Após tricotomia na região cervical e inguinal direitas, cada animal foi colocado na mesa cirúrgica em posição supina e a indução anestésica foi feita com tiopental sódico (Thionembutal - Abbot - São Paulo - SP), na dose de $5 \mathrm{mg} / \mathrm{kg}$ intravenosa, pela veia jugular externa esquerda. Para intubação usouse cânula traqueal (Rüsch - Alemanha) de calibres variando de 7 a 8,5F, de acordo com o diâmetro da traquéia. Empregou-se também sonda nasogástrica de polietineno $20 \mathrm{~F}$ (Sondoplast - São Paulo -SP).

A artéria e a veia femoral direita foram dissecadas e cateterizadas com cânulas de polietileno 8F (Sondoplast - São Paulo - SP). 0 cateter arterial foi utilizado para coleta de amostras de sangue para dosagem gasométrica e monitorização da pressão arterial, usando-se um manômetro de vidro (Vidrolex - São Paulo - SP) com mercúrio. A incoagulabilidade do sangue no cateter foi obtida com solução de Ringer com lactato de sódio, contendo 0,5 unidade de heparina (Liquemine - Roche - São Paulo - SP) para cada $\mathrm{ml}$ da solução. 0 cateter venoso foi utilizado na coleta de amostras de sangue para avaliação bioquímica e dos elementos sangüíneos, além de infusão de drogas (heparina e tiopental).

A ventilação foi no modo controlado, com 0 respirador a volume London SW2 (CF Palmer LTD - Londres - Inglaterra), com volume corrente de $14 \mathrm{ml} / \mathrm{kg}$, freqüência de 16 ciclos por minuto, FiO de $21 \%$ e pressão expiratória final positiva (PEEP) de $3 \mathrm{~cm}$ de água.

Foi feita incisão obliqua no bordo superior do músculo esternocleidomastóideo, identificando-se a veia jugular interna e a artéria carótida. Aplicou-se $100 \mathrm{Ul} / \mathrm{kg}$ de peso de heparina, por via intravenosa. Nos dois primeiros casos, a anastomose ou o by-pass foi venovenosa, utilizando-se para drenagem a veia jugular interna e para a reinfusão a veia femoral esquerda. Nos nove casos restantes, o by-pass foi veno-arterial. Nesses casos, a artéria carótida foi ligada distalmente, cateterizando-se a porção proximal com uma cânula de polietileno (CPL Medical's - São Paulo -SP), do maior calibre possível, a qual foi introduzida até atingir a raiz da aorta. Nos nove casos de by-pass veno-arterial foram utilizados para reinfusão cateteres de calibre $16 \mathrm{~F}$, e nos dois casos de by-pass venovenoso, calibre $12 \mathrm{~F}$. Nos seis últimos experimentos, a veia jugular teve canulada tanto sua porção proximal quanto a distal ou cefálica, sendo que o cateter proximal foi conduzido até o átrio direito. Essa mudança de abordagem visou melhorar a drenagem venosa.

Os cateteres de drenagem foram conectados com auxílio de um tubo de acrílico em forma de Y (Braile - Biomédica - São José do Rio Preto SP) a um outro com diâmetro interno de $3 / 8$ de polegada, para condução da drenagem venosa ao oxigenador.

Nos cinco primeiros animais, o prime do 
circuito extracorpóreo foi feito com solução de Ringer com lactato de sódio (Ringer - Lactato Glicolabor - Ribeirão Preto - SP) e solução de gelatina a 3,5\% (Hisocel - Campinas - SP), na proporção de $2: 1$, no total de $750 \mathrm{ml}$. No sexto animal foi diminuído o volume do prime para $450 \mathrm{ml}$, sendo $250 \mathrm{ml}$ de solução de Ringer com lactato de sódio e $200 \mathrm{ml}$ de solução de gelatina a $3,5 \%$. Nos cinco últimos experimentos (casos 7 a 11 ), empregou-se $250 \mathrm{ml}$ de sangue fresco total anticoagulado com citrato de sódio e $200 \mathrm{ml}$ de Ringer com lactato de sódio.

Cinco minutos antes do início da ECMO, visando simular as condições clínicas de hipóxia, foi feita redução da freqüência respiratória para seis ciclos por minuto, mantendo-se os demais parâmetros, e colhida gasometria arterial. Essas condições permaneceram até o final do experimento.

Foi então iniciada a ECMO propriamente dita. $O$ sangue venoso drenado foi impulsionado com auxílio de uma bomba de rolete (BraileBiomédica - São José do Rio Preto - SP), para um oxigenador de membrana microporosa, com poros de 0,03 ìm de diâmetro e $0,7 \mathrm{~m}^{2}$ de superfície total efetiva de troca gasosa, acoplado a um sistema permutador de calor (BraileBiomédica - São José do Rio Preto - SP) para manter a temperatura corporal. O sangue arterializado foi reinfundido na raiz da aorta, via carótida, por um cateter de polietileno 16F ( 9 casos), ou na veia cava inferior, via femoral ( 2 casos), usando-se cateteres $12 \mathrm{~F}$. 0 complexo extracorpóreo, com acesso para coleta de amostras de sangue tanto do retorno venoso como da reinfusão arterial, permitiu a realização de gasometrias conforme o protocolo. Amostras de sangue para outras dosagens foram colhidas também da artéria ou da veia femoral.

A manutenção foi feita com fluxo de 45 a $170 \mathrm{ml} / \mathrm{kg} / \mathrm{min}(104,1 \pm 35,3 \mathrm{ml} / \mathrm{kg} / \mathrm{min})$. Sempre que necessário, o volume do perfusato no reservatório foi mantido em condições de segurança com auxílio de solução de Ringer com lactato de sódio, solução de cloreto de sódio a $0,9 \%$ (Ibifan - Belém -PA) ou sangue fresco total homólogo. Procurou-se manter o tempo de coagulação ativado entre 180 e 360 segundos com uso de heparina intravenosa na dose de $20 \mathrm{Ul} / \mathrm{kg}$ de peso, a cada hora, até o fim do experimento, omitindo seu uso caso o tempo de coagulação ativado estivesse acima do valor limite superior.

Foram colhidas amostras de sangue para avaliações bioquímica e hematimétrica no pré, após 6 e 12 horas de ECMO. O s exames realizados foram: hematócrito, hemoglobina, leucócitos, plaquetas, albumina e globulina.Para as dosagens hematimétricas foi utilizado o aparelho Coulter T890 (Coulter - Hialeah - EEUU).

Os animais receberam cuidados de acordo com as recomendações do Committee on Careand Use of Laboratory Animals of the Institute of Laboratory Animal Resources - National Research Council.

Ao final de cada experimento, o animal foi sacrificado por infusão de tiopental sódico, seguida de drenagem total do sangue.

Para fins de análise foram considerados dois grupos: 0 Grupo 1 incluiu os primeiros seis animais, onde a reposição hídrica foi liberal e não foi utilizado sangue no prime e o Grupo 2, com os cinco últimos animais, onde a reposição volêmica e a drenagem venosa foram, supostamente, mais adequadas e se utilizou sangue homólogo, no prime.

No estudo estatístico foram empregados os testes de Mann-Whitney e Análise da Variância e, no caso deste último demonstrar variação significativa $(p<0,05)$, aplicou-se 0 teste de Tukey.

\section{Resultados}

Nesses experimentos, verificou-se que o valor médio do hematócrito pré-ECMO, que era de $28,0 \%$, reduziu-se para $12,2 \%$ nos que atingiram 6 horas de experimento, ficando próximo desse valor nos que chegaram a 12 horas (15\%), conforme tabela 1. Já no segundo grupo, o hematócrito médio pré-ECMO que era de $29,8 \%$, manteve-se estável com 6 e 12 horas (29,6\% e $28 \%$, respectivamente) conforme tabela 2. A análise comparativa entre os Grupos 1 e 2 mostra que houve diferença estatisticamente significativa entre eles, com 6 horas $(p=0,01)$ e 12 horas de evolução $(p=0,04)$ conforme figura 1 . 
Tabela 1 Valores hematimétricos no Grupo 1, no pré, com 6 e 12 horas de ECMO

\begin{tabular}{|l|l|l|l|}
\hline Testes & Pré-ECMO & 6 horas & 12 horas \\
\hline Hematócrito (\%) & 28 & 12,2 & 15 \\
\hline Hemoglobina (g\%) & 9,3 & 3,9 & 5,0 \\
\hline Leucócitos (/I) & $5,95 \times 10^{9}$ & $1,94 \times 10^{9}$ & $3,9 \times 10^{9}$ \\
\hline Plaquetas (/l) & $430 \times 10^{9}$ & $194 \times 10^{9}$ & $240 \times 10^{9}$ \\
\hline Albumina (g\%) & 2,5 & 0,7 & -- \\
\hline Globulina (g\%) & 2,5 & 1,4 & -- \\
\hline Prot.Totais (g\%) & 5,0 & 2,1 & - \\
\hline
\end{tabular}

Prot. Totais $=$ proteínas totais

Tabela 2. Valores hematimétricos no Grupo 2, no pré, com 6 e 12 horas de ECMO

\begin{tabular}{|l|l|l|l|}
\hline Testes & PréECMO & 6 horas & 12 horas \\
\hline Hematócrito (\%) & 29,8 & 29,6 & 28 \\
\hline Hemoglobina (g\%) & 9,8 & 9,8 & 9,3 \\
\hline Leucócitos (/I) & $8,1 \times 10^{9}$ & $7,84 \times 10^{9}$ & $7,18 \times 10^{9}$ \\
\hline Plaquetas (/I) & $474 \times 10^{9}$ & $302 \times 10^{9}$ & $220 \times 10^{9}$ \\
\hline Albumina (g\%) & 2,7 & 2,5 & 2,2 \\
\hline Globulina (g\%) & 2,2 & 2,4 & 2,1 \\
\hline Prot.Totais (g\%) & 4,9 & 4,9 & 4,3 \\
\hline
\end{tabular}

Prot.Totais= proteínas totais

Q uanto à albumina e globulina, observou-se reduções importantes ao longo do experimento, conforme tabelas 1e 2. Após 6 horas de ECMO, porém, o valor médio do Grupo 1 reduziu-se a $0,7 \mathrm{~g} \%$, tendo havido 1 óbito, enquanto que no Grupo 2, ficou bem próximo da média préoperatória, com $2,46 g \%(p=0,04)$.

0 número médio de leucócitos no pré-ECMO foi de $6,927 \times 10^{9}$ leucócitos/L para o grupo total, ficando em 4,89 × $10^{9}$ leucócitos/L com 6 horas de ECMO e 5,95 × $10^{9}$ leucócitos/L com 12 horas. 0 Grupo 1 apresentava 5,95 × $10^{9}$ leucócitos/L no pré-ECMO , 1,94 x $10^{9}$ leucócitos/L com 6 horas e $3,900 \times 10^{9}$ leucócitos/L com 12 horas. 0 Grupo 2 apresentava 8,100 × $10^{9}$ leucócitos/L no pré-ECMO,

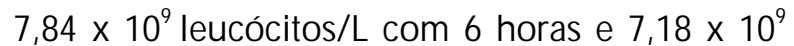
leucócitos/L com 12 horas. Comparando-se 0 Grupo 1 com o Grupo 2 verifica-se diferença significativa com 6 horas $(p=0,01)$ e 12 horas de ECMO $(p=0,04)$, figura 2 .

As plaquetas também sofreram redução ao longo do tempo, verificando-se que a quantidade média pré-ECMO foi, para o grupo total, de $450 \mathrm{x}$ $10^{9} /$, tendo sido reduzida para $248 \times 10^{9} / / \operatorname{com} 6$ horas e para $227 \times 10^{9} / /$ com 12 horas, tabelas $1 \mathrm{e}$ 2. A redução, portanto, foi de $45 \%$ em 6 horas e de $50 \%$ em 12 horas, figura 3. Não ocorreram diferenças significativas quando se comparou 0 Grupo 1 ao Grupo 2 ( $p=0,65$ no pré; $p=0,68$ com 6 horas; $p=0,29$ com 12 horas). 


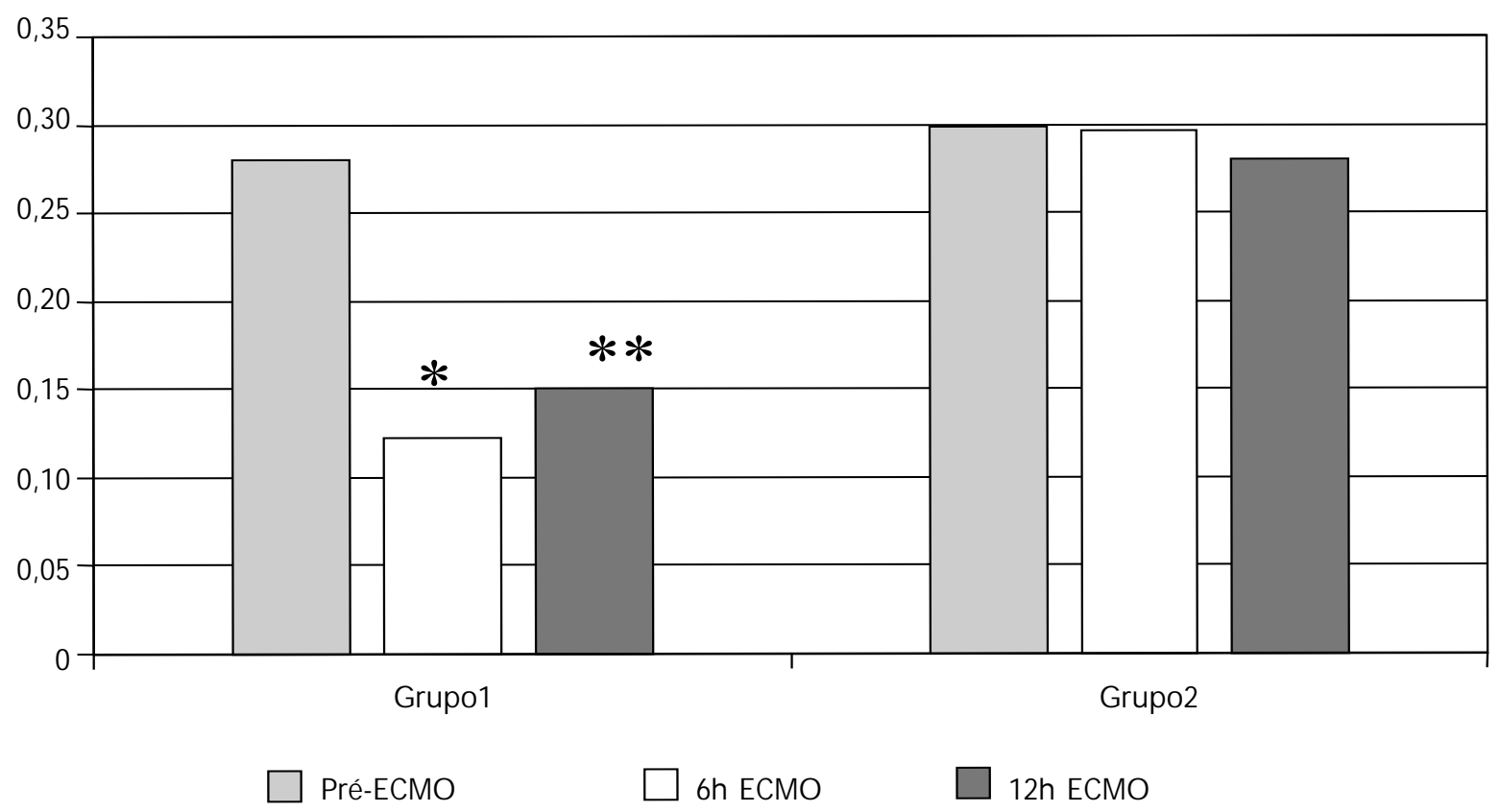

Figura 1. Valores médios do hematócrito em porcentagem, nos Grupos 1 e 2

Nota-se diferença significativa entre os Grupos 1 e 2 com 6 horas $(* p=0,01)$ e 12 horas $(* x=0,04)$ de ECMO.

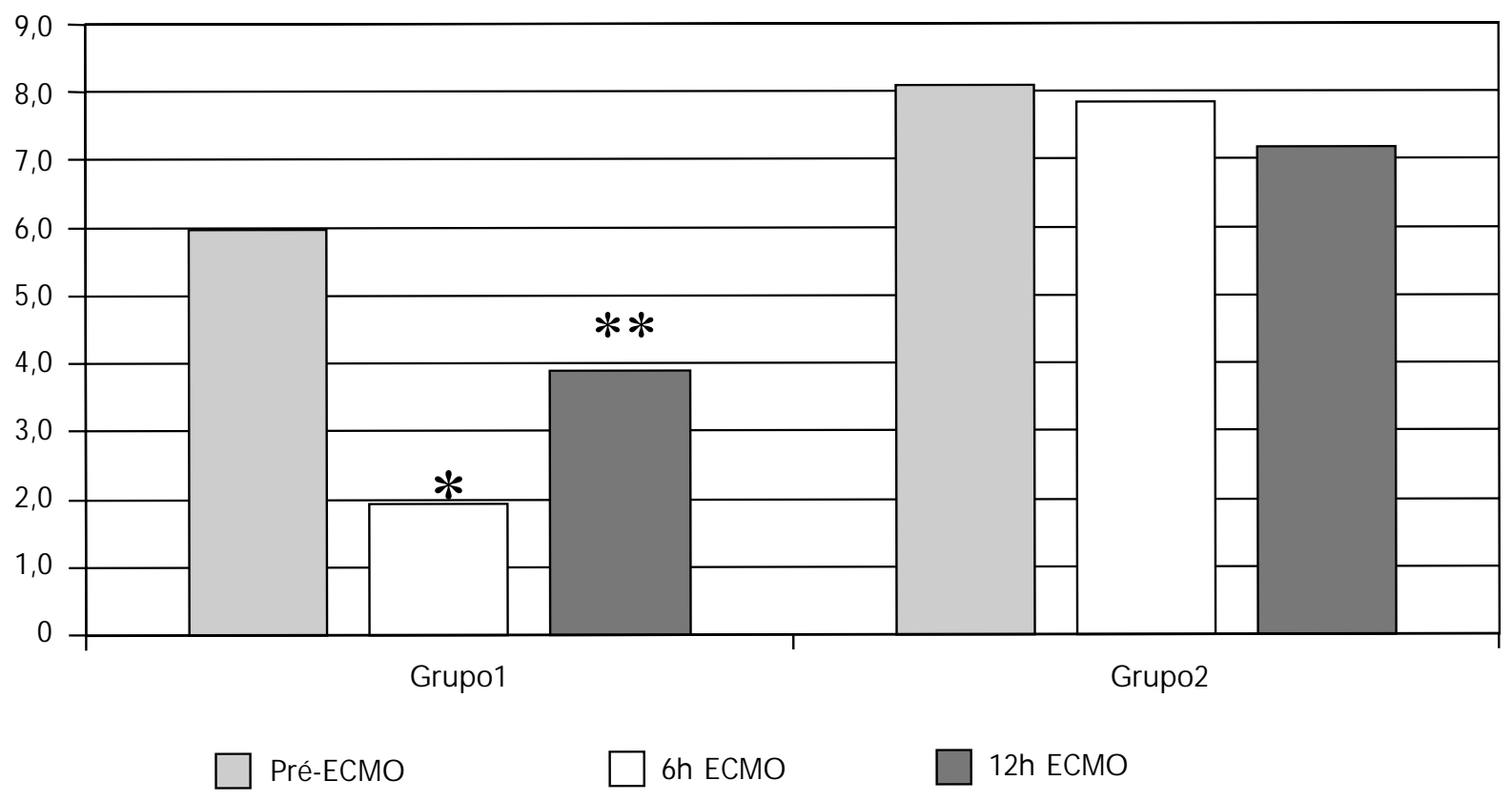

Figura 2. Valores médios de leu cócitos, $\times 10^{9} / \mathrm{dL}$, nos Grupos 1 e 2 Nota-se diferença significativa entre os Grupos 1 e 2 com 6 horas $(* p=0,01)$ e 12 horas $\left({ }^{* *} p=0,04\right)$ de ECMO 


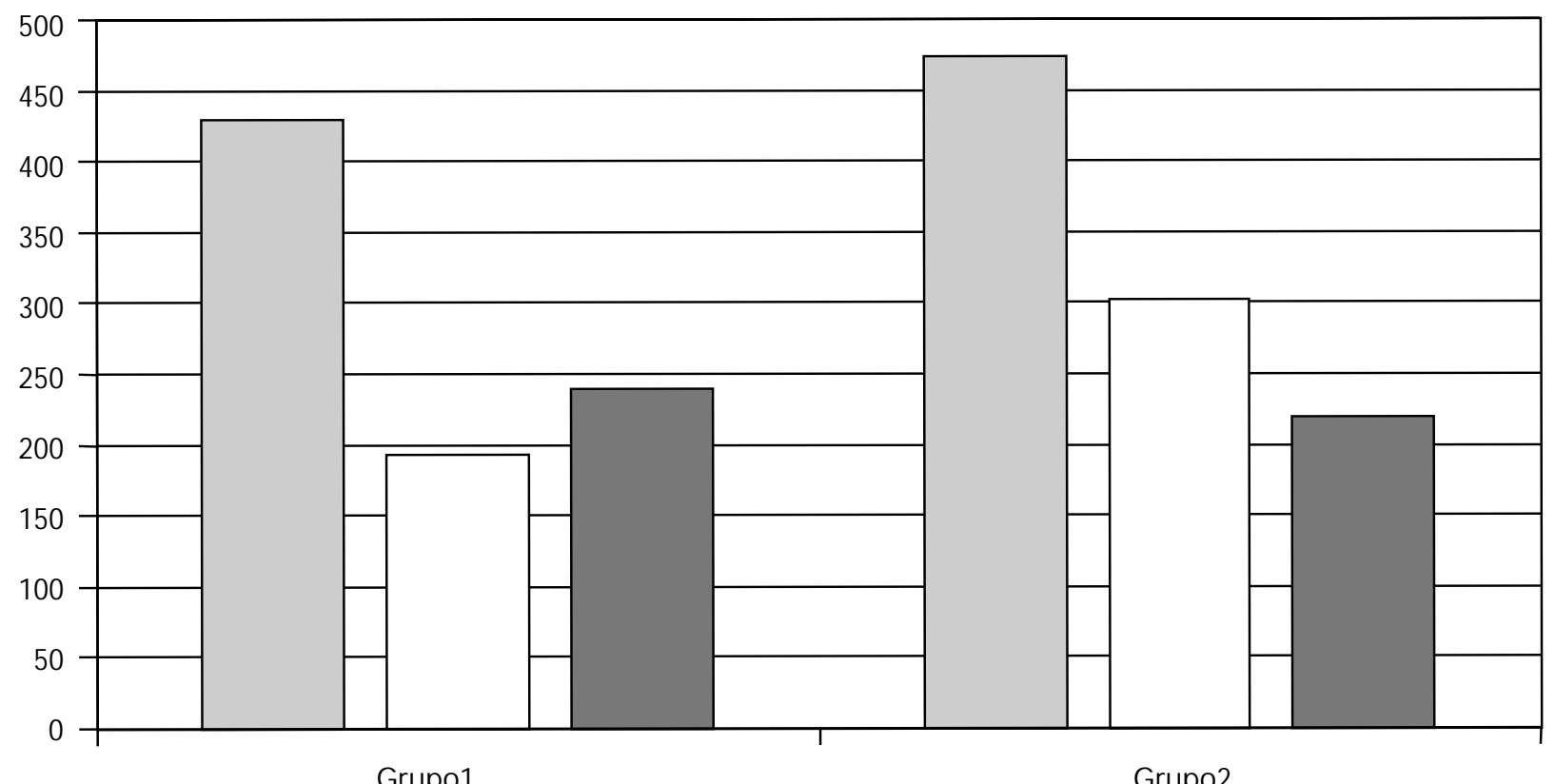

Pré-ECMO

6h ECMO

12h ECMO

Figura 3. Valores médios de plaquetas, $\times 10^{9} /$, nos Grupos 1 e 2

\section{D iscussão}

0 presente estudo detectou interferências na administração de líquidos sobre a hematimetria. Nos seis primeiros casos não se utilizou sangue na composição do prime. Este fato, aliado ao excesso de líquidos infundidos, provocou queda acentuada nos níveis de hematócrito e hemoglobina, com as conseqüências inerentes. A análise comparativa entre os Grupos 1 e 2 mostra que houve diferença estatisticamente significativa entre eles, com 6 horas $(p=0,01)$ e 12 horas de evolução $(p=0,04)$.

A hiper-hidratação ocorreu neste estudo, fato este que foi bem documentado pela queda do hematócrito e pelas alterações anatomopatológicas. Teve como causa a reposição inadvertida de líquidos, na tentativa de manter um fluxo de infusão elevado, não compatível com o grau de retorno venoso.

Essas evidências levaram à utilização de dupla via de drenagem da veia jugular interna (coto proximal e distal) para aumentar o retorno venoso, conforme preconizado por O'Connor et al.(1993). Esse procedimento e a utilização de sangue no prime foram fatores capitais na boa evolução do Grupo 2 .

0 estudo demonstra, portanto, a necessidade de boa drenagem venosa que permita fluxo adequado, sem complementação volêmica excessiva. Nos casos que já apresentarem sinais compatíveis com hiper-hidratação, seria indicada a utilização de ultrafiltração, de acordo com as normas propostas por Elliot (1990).

Quanto à albumina e globulina, observou-se reduções importantes ao longo do experimento. Verifica-se que o nível médio pré-operatório esteve dentro do valor esperado para a espécie (albumina normal em ovinos $=2,96 \mathrm{~g} \%$ ) (11), já que no Grupo 1 (dosagem em 4 casos), o valor médio foi $2,57 \mathrm{~g} \%$ e no Grupo 2 (dosagem em 5 casos), foi de 2,68g\% $(p=0,80)$. Após 6 horas de ECMO, porém, o valor médio do Grupo 1 reduziu-se a $0,7 \mathrm{~g} \%$, tendo havido 1 óbito, enquanto que no Grupo 2, ficou bem próximo da média pré-operatória, com $2,46 \mathrm{~g} \%$ $(p=0,04)$. Por fim, com 12 horas de ECMO, o valor médio da albumina no Grupo 1 não pôde ser aferido, 
uma vez que foi feita dosagem em apenas 1 animal (em 2 não foi realizada e 3 foram a óbito antes de 12 horas). No Grupo 2, a média estava em 2,22 g\%, e não houve óbitos até 12 horas de ECMO. Os valores médios da globulina total (globulina normal em ovinos $=2,85 \mathrm{~g} \%$ ) (8) acompanharam a evolução dos valores da albumina, em ambos os grupos. Mais uma vez, a hemodiluição deve ser considerada como a causa da queda progressiva dos valores.

Desde os primórdios da circulação extracorpórea, os pesquisadores têm se preocupado com a ocorrência de trombocitopenia, edema, sangramento, hemólise e falência de órgãos. $A$ análise crítica dos dados hematimétricos do presente estudo mostra queda acentuada dos níveis de hematócrito e hemoglobina. Ressalte-se que verificaram, em pacientes submetidos à ECMO, que o aumento no nível de hemoglobina livre ocorre mais acentuadamente após 48 horas, concomitantemente com 0 aumento da dosagem dos produtos de degradação da fibrina e a presença de coágulos no circuito, além de diminuição nos níveis de fibrinogênio (9). Embora no presente estudo não tenham sido dosados fibrinogênio, produtos degradados de fibrina e hemoglobina livre, é bem provável que não fossem encontradas alterações, em virtude de serem fenômenos tardios e os experimentos terem sido encerrados após 12 horas de evolução.

Em relação aos leucócitos foi observada a redução destes quando se compara o Grupo $1 \mathrm{com}$ - Grupo 2, onde se verifica uma diferença significativa com 6 horas $(p=0,01)$ e 12 horas de ECMO $(p=0,04)$.

$A$ redução dos leucócitos durante a ECMO é observada na literatura $(7,8)$, porém a sua capacidade de fagocitose e de destruição intracelular não estão alteradas (9).

As plaquetas também sofreram redução ao longo do tempo, verificando-se que a quantidade média pré-ECMO foi, para o grupo total, de $450 \mathrm{x}$ $10^{9} / \mathrm{l}$, tendo sido reduzida para $248 \times 10^{9} / \mathrm{l}$ com 6 horas e para $227,5 \times 10^{9} / 1$ com 12 horas. A redução, portanto, foi de $45 \%$ em 6 horas e de $50 \%$ em 12 horas. Não ocorreram diferenças significativas quando se comparou o Grupo 1 ao Grupo 2 ( $p=0,65$ no pré; $p=0,68$ com 6 horas; $p=0,29$ com 12 horas).

Em um dos estudos na literatura verificaram em 10 recém-nascidos, 15 minutos após o início da ECMO, diminuição média de $26 \%$ no número de plaquetas e de mais 16\% após 1 hora, perfazendo um total de $42 \%$ (12). 0 estudo para verificação da função plaquetária aos 15 minutos de estudo mostrou que, em média, $46 \%$ das plaquetas tiveram sua resposta ao colágeno diminuída e significante diminuição da resposta à ristocetina e à adenosina5'-difosfato. Documentaram também redução significante na liberação de adenosina-trifosfato plaquetária. A reposição com infusão plaquetária mostrou elevação apenas transitória, com retorno aos valores pré-transfusionais após cerca de 1 hora. Nos ovinos ora estudados, como visto, também se observou redução significativa na contagem plaquetária, com 6 e 12 horas de ECMO.

\section{Conclusões}

A oxigenação extracorpórea por membrana é uma técnica de suporte cardiopulmonar prolongado que apresenta várias complicações e dentre elas as alterações hematológicas. A identificação dos fatores que interferem nestes mecanismos permitem amenizar essas agressões, reduzindo as suas complicações.

Um desses fatores identificados no presente estudo foi a oferta excessiva de volume que levou à alterações hematimétricas.

\section{Extra-corporeal membrane oxygenation and hematological alterations in an experimental study}

Aírton C. Moscardini, Moacir F. Godoy, Domingos M. Braile, José M. P. de Godoy, Marcelo J. Soares, Antonio C. Brandi, Serginando L. Ramin

\section{Abstract}

Extra-corporeal membrane oxygenation is a technique of prolonged cardiopulmonary support, with the objective of assisting the lungs and/or heart when they start to fail and do not respond to conventional non-invasive treatment. The aim of this stu dy wasto evaluate hematological alterations during 12 hours of extra-corporeal membrane oxygenation in animals.

Eleven sheep, six males and five females, of the Santa 
Inês breed with weights ranging from 5.4 to $15 \mathrm{~kg}$ $(12.2 \pm 3.1 \mathrm{~kg})$ werestudied. With thefirst fiveanimals, Ringer solution with sodium lactate(Ringer - Lactato Glicolabor - Ribeirão Preto - SP) and a 3.5\% gelatine solution (Hisocel - Campinas-SP) wereused to prime the extra-corporeal circuit in the proportion of $2: 1$ making a total of $750 \mathrm{ml}$. In the sixth animal the volume of the circuit primer was reduced to $450 \mathrm{ml}$, composed of $250 \mathrm{ml}$ of Ringer solution with sodium lactate and $200 \mathrm{ml}$ of $3.5 \%$ gelatine solution. In the other 5 animals $250 \mathrm{ml}$ of total fresh blood anticoagulated with sodium citrate and $200 \mathrm{ml}$ of Ringer solution with sodium lactate were employed. The blood tests performed were: hematocrit, haemoglobin, leukocytes, platelets, albumin and globulin. Mann-Whitney and variation analysis tests were used for statistical evaluation and, in the case that the variation analysis gave a significant difference $(p<0.05)$, the Tukey test was applied. A reduction of all the elements analysed wasobserved with a significant statistical difference in the values of hematocrit and leukocytes between the two groups.

The hematological alterations seen in thisstu dy were exclusively dueto theexcessivesupply of fluid volumes. Rev.bras.hematol.hemoter.,2002,24(2):97-104

Keywords: Extra-corporeal membrane oxygenation, haematological alterations

\section{Referências bibliográficas}

1. Pagani FD, Aaronson KD, Swaniker F, Bartlett $\mathrm{RH}$. The use of extracorporeal life support in adult patients with primary cardiac failureas a bridge to implant left ventricular assist devise. Ann Thorac Surg 2001; 71(3): S77-85.

2. WeberTR, Connors RH, Tracy JrTF, Bailey PV, Stephens C, Keenan W. Prognostic determinants in extracorporeal membrane oxygenation for respiratory failure in newborns. Ann. Thorac. Surg New York, 1990; 50:720-3.

3. Kawahito S, Maeda T, Yoshikawa M, Takano T, Nonaka K, Linneweber J, Mikami M, Motomura T, Ichikawa S, Glueck J, Nose Y.
Blood trauma induced by clinically accepted oxigenators. ASIO J 2001; 47(5): 492-5.

4. Steinhorn RH, Isham-Schopt B, Smith C, Green TP. Hemolysis during long-term extracorporeal membrane oygenation. J Pediatric 1990; 116(^6): 1005-7.

5. Graulich J, Walzog B, Marcinkow ski M, Bauer K, Kossel H, Fuhrmann G, Buhrer C, Gaehtgens $P$, Versmold HT. Leukocyte and endothelial activation in a laboratory model of extracorporeal membrane oxigenation (ECMO). Pediatr Res 2000; 48(5):679-84.

6. Adrian K, Mellgren K, Skogby M, Friberg LG, Mellgren $G$, Wadenvik $H$. Cytokine release during long-term extracorporeal circulation in an experimental model. Artif Organs 1998; 22(10):859-63.

7. Hocker JR, Wellhausen SR, Ward RA, Simpson PM, Cook LN. Effect of extracorporeal membrane oxygenation on leucocyte function in neonates. Artif. Organs, Cleveland, 1991; 15:23-28.

8. Bergman P, Belboul A, Friberg LG, AL-Khaja $N$, Mellgren $G$, Roberts $D$. The effect of prolonged perfusion with a membrane oxygenator (PPMO) on white blood cells. Perfusion, New York, 9(1): 35-40, 1994.

9. De Puydt LE, Schuit KE, Smith SD. Effect of extracorporeal membrane oxygenation on neutrophil function in neonates. Crit. Care M ed., Baltimore, 21(9): 1324-1327, 1993.

10. O'Connor TA, Haney BM, Grist GE, Egelhoff JC, Snynder CL, Ashcraft KW. Decreased incidence of intracranial hemorrhageusing cephalicjugular venousdrainage during neonatal extracorporeal membrane oxigenation. J. Pediatr. Surg. New York, 28 (10): 1332-1335, 1993.

11. Elliot MJ. Perfusion for pediatric open heart surgery. Sem. Thorac. Cardiovasc. Surg. New York, 2(4): 332-340, 1990.

12. Robinson TM, Kickler TS, Walker LK, Ness $P$, Bell W. Effect of extracorporeal membrane oxygenation on platelets in newborns. Crit. C are M ed. Baltimore, 21(7): 1029-1034, 1993.

Recebido: 24/02/2002

Aceito: $28 / 03 / 2002$ 